\title{
Simulação Numérica e Método Constructal Design Aplicados à Avaliação Geométrica de um Trocador de Calor Solo-Ar com Formato T
}

\section{Numerical Simulation and Constructal Design Applied to the Geometric Evaluation of a T-Shaped Earth-Air Heat Exchanger}

Gerusa Camargo Rodrigues ${ }^{1}$, Michel Kepes Rodrigues ${ }^{2}$, Luiz Alberto Oliveira Rocha $^{3}$, Elizaldo Domingues dos Santos ${ }^{4}$, Emanuel da Silva Diaz Estrada ${ }^{5}$, Liércio André Isoldi6

\section{RESUMO}

O Trocador de Calor Solo-Ar (TCSA) é um dispositivo constituído por um ou mais dutos enterrados no solo, por onde o ar é forçado a escoar. No processo de troca de calor entre ar e solo, o ar sai do TCSA com uma temperatura mais amena em relação à sua temperatura de entrada, melhorando a condição térmica de edificações. A partir de um TCSA com duto reto (uma entrada e uma saída) adotado como referência, diferentes configurações geométricas de um TCSA com formato T (uma entrada e duas saídas) foram propostas através do método Constructal Design. O grau de liberdade considerado relaciona 0 comprimento do ramo bifurcado com 0 comprimento do ramo principal $\left(L_{1} / L_{0}\right)$. Simulações numéricas realizadas no software FLUENT, baseado no Método dos Volumes Finitos (MVF), permitiram avaliar o potencial térmico do TCSA, enquanto a perda de carga e o volume de solo foram determinados analiticamente. Os resultados indicaram que a instalação definida por $L_{1} / L_{0}=0,1$ maximiza o potencial térmico; a instalação definida por $L_{1} / L_{0}=7,0$ minimiza o volume de solo ocupado e a perda de carga do escoamento; e ao considerar os três parâmetros simultaneamente, a instalação definida por $L_{1} / L_{0}=$ 7,0 apresenta melhor desempenho.

Palavras-chave: Trocador de Calor Solo-Ar (TCSA). Constructal Design. Modelagem Computacional. Potencial térmico. Perda de carga.

\section{ABSTRACT}

The Earth-Air Heat Exchanger (EAHE) is a device consisting of one or more buried ducts in the soil, through which the air is forced to flow. In the heat exchange process between air and soil, the air leaves the EAHE with a milder temperature relative to its inlet temperature, improving the thermal condition of buildings. From an EAHE with a straight duct (with one inlet and one outlet) adopted as reference, different geometric configurations of a T-shaped EAHE (with one inlet and two outlets) were proposed by means the Constructal Design method. The degree of freedom considered relates the length of the bifurcated branch to the length of the main branch $\left(L_{1} / L_{0}\right)$. Numerical simulations performed in the FLUENT software, based on the Finite Volume Method (FVM), allowed to evaluate the thermal potential of the EAHE, while the pressure drop and soil volume were analytically determined. The results indicated that the installation defined by $L_{1} / L_{0}=0.1$ maximizes the thermal potential; the installation defined by $L_{1} / L_{0}=7.0$ minimizes the occupied soil volume and the pressure drop; and considering the three parameters simultaneously, the installation defined by $L_{1} / L_{0}=$ 7.0 has the best performance.

Keywords: Earth-Air Heat Exchanger (EAHE), Constructal Design. Computational Modeling. Thermal potential. Pressure drop.
${ }^{1}$ Mestranda em Modelagem Computacional, Universidade Federal do Rio Grande - FURG.

E-mail: gerusa.cr@gmail.com

${ }^{2}$ Mestre em Modelagem Computacional, Universidade Federal do Rio Grande - FURG.

${ }^{3}$ Doutor em Engenharia Mecânica, Universidade do Vale do Rio dos Sinos - UNISINOS.

${ }^{4}$ Doutor em Engenharia Mecânica, Universidade Federal do Rio Grande - FURG.

${ }^{5}$ Doutor em Engenharia Mecânica, Universidade Federal do Rio Grande - FURG.

${ }^{6}$ Doutor em Engenharia Mecânica, Universidade Federal do Rio Grande - FURG. 


\section{INTRODUÇAOO}

O esgotamento das fontes convencionais de energia, aliado à preocupação sobre o meio ambiente, desencadeou uma crescente busca por fontes alternativas e renováveis de energia. Sabe-se que o solo é um grande reservatório de energia térmica, sendo essa energia térmica derivada da radiação solar que é a principal fonte de energia renovável existente (SEN, 2008). Segundo Vaz et al. (2011), o aproveitamento dessa energia térmica existente no solo pode ser feito através de dispositivos conhecidos como Trocadores de Calor Solo-Ar (TCSA).

O TCSA é um equipamento constituído por um ou mais dutos enterrados no solo, através dos quais o ar é forçado a escoar, por exemplo, com o auxílio de ventiladores. $\mathrm{O}$ solo absorve (em períodos quentes) e cede (em períodos frios) calor para o ar que escoa através do TCSA. Assim, o ar que sai do TCSA possui temperatura mais amena em relação à sua temperatura de entrada. Esse ar que sai do TCSA é então usado para a melhoria da condição térmica de ambientes construídos, permitindo uma redução significativa no consumo de energia elétrica com condicionadores de ar na busca do conforto térmico (VAZ, 2011; VAZ et al., 2011; VAZ et al., 2014).

Estudos mais recentes realizados a partir de análises experimentais e numéricas sobre o funcionamento do TCSA podem ser encontradas em Bansal et al. (2009), Vaz et al. (2011), Bisonyia et al. (2013) e Vaz et al. (2014).

Análises numéricas a respeito da influência de parâmetros como diâmetro e comprimento dos dutos, velocidade do ar na entrada do TCSA, umidade do ar e perda de carga, entre outros, podem ser observados em Trzaski e Zawada (2011), Benhammou e Draoui (2015), Niu et al. (2015), Belatrache et al. (2016) e Amanowicz (2018).

Além disso, o método Constructal Design vem sendo empregado em processos de avaliação geométrica de sistemas de fluxo, principalmente em problemas de transferência de calor e mecânica dos fluidos. O Constructal Design é um método que considera um ou mais parâmetros de performance, restrições e graus de liberdade, a fim de avaliar a influência da configuração geométrica no desempenho de um determinado sistema de engenharia. Este método de avaliação geométrica é baseado na Lei Constructal desenvolvida por Bejan (1996) que, simplificadamente, determina que os sistemas de fluxo devem evoluir com o tempo, adquirindo melhores configurações para fornecer mais acesso às correntes que fluem através deles. Aplicações do método Constructal Design 
em instalações de TCSA podem ser encontradas em Rodrigues (2014), Rodrigues et al. (2015) e Brum (2016).

Diferentemente dos trabalhos apresentados, o presente estudo propõe uma nova configuração geométrica para o TCSA, de modo que seja possível atender duas edificações ou dois cômodos de uma edificação simultaneamente. Trata-se de instalações com arranjo dos dutos em forma de T, como mostrado na Figura 1, com uma entrada e duas saídas. O método Constructal Design é aplicado na avaliação geométrica do TCSA, enquanto que a otimização geométrica é realizada através da técnica de Busca Exaustiva.

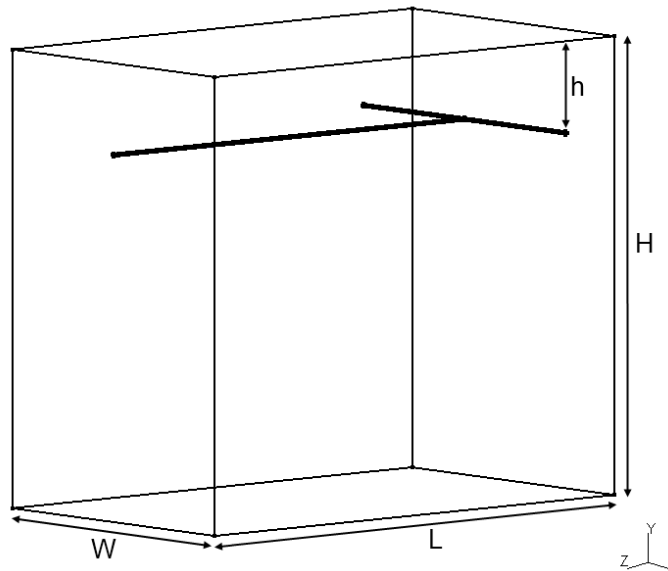

(a)

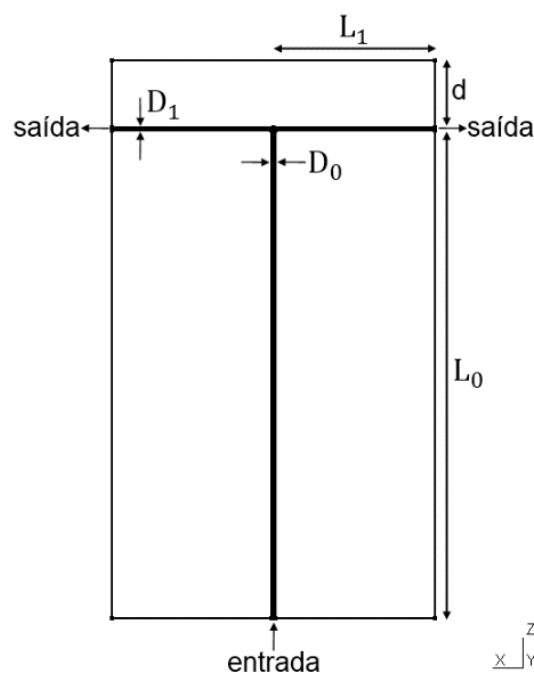

(b)

Figura 1. (a) Vista em perspectiva do domínio computacional; (b) Vista superior do domínio computacional.

Deste modo, o principal objetivo deste trabalho é analisar numericamente o desempenho térmico e analiticamente o desempenho fluidodinâmico das instalações, avaliando a influência dos comprimentos dos dutos que formam a geometria, definidos pela razão $L_{1} / L_{0}$, no volume de solo ocupado, no potencial térmico e na perda de carga do TCSA. Cabe destacar que o método Design Constructal foi adotado para a determinação de configurações geométricas possíveis, respeitando as restrições e os graus de liberdade impostos, ou seja, ele foi usado para a definição do espaço de busca. Então, através da técnica de Busca Exaustiva, onde todas as configurações geométricas propostas tem seus resultados (numéricos ou analíticos) comparadas entre si, uma otimização geométrica foi realizada. 


\section{DESCRIÇAO DO PROBLEMA}

O domínio computacional utilizado para a análise numérica do problema é representado na Figura 1. Como explicado, trata-se de um TCSA cuja configuração geométrica consiste em um arranjo de dutos na forma de $\mathrm{T}$.

Baseado em Vaz et al. (2011) e Brum et al. (2012), como condições de contorno tem-se que as faces laterais e a face inferior da porção de solo são isoladas termicamente enquanto que a face superior representa a superfície do solo e tem temperatura prescrita dada a partir de uma função periódica ajustada estatisticamente com base em dados experimentais. Na entrada do duto foi adotada a velocidade de escoamento do ar de $3,3 \mathrm{~m} / \mathrm{s}$ e temperatura prescrita também dada por função periódica ajustada a partir de dados experimentais. Nas saídas do TCSA foi adotada como sendo a pressão atmosférica.

As propriedades dos materiais considerados nas simulações numéricas foram definidas de acordo com a Tabela 1.

Tabela 1. Propriedades dos materiais.

\begin{tabular}{cccc}
\hline Componentes & $\boldsymbol{\rho}\left(\mathbf{k g} / \mathbf{m}^{3}\right)$ & $\boldsymbol{k}(\mathbf{W} / \mathbf{m} \cdot \mathbf{K})$ & $\boldsymbol{c}_{\boldsymbol{p}}(\mathbf{J} / \mathbf{k g} \cdot \mathbf{K})$ \\
\hline Solo argiloso & 1800 & 2,1 & 1780 \\
$\mathrm{Ar}$ & 1,16 & 0,0242 & 1010 \\
\hline
\end{tabular}

Sendo na Tabela 1: $\rho$ a massa específica, $k$ a condutividade térmica e $c_{p}$ o calor específico.

Como Instalação Referência (Figura 2) adotou-se uma instalação de TCSA com um duto reto de $30 \mathrm{~m}$ de comprimento e com diâmetro de $110 \mathrm{~mm}$, cujo modelo computacional foi verificado com base no estudo apresentado em Nunes (2015). As dimensões para o solo desta instalação foram consideradas como sendo de $30 \mathrm{~m}$ de comprimento, $15 \mathrm{~m}$ de altura e $5 \mathrm{~m}$ de largura.

Os dutos foram enterrados a uma profundidade $(h)$ de $3 \mathrm{~m}$, conforme estudo de Brum et al. (2012); e manteve-se uma distância (d) de $2 \mathrm{~m}$ entre os dutos e as paredes laterais do solo para evitar que a condição de contorno das paredes influencie nos resultados, conforme estudo de Rodrigues et al. (2015). 


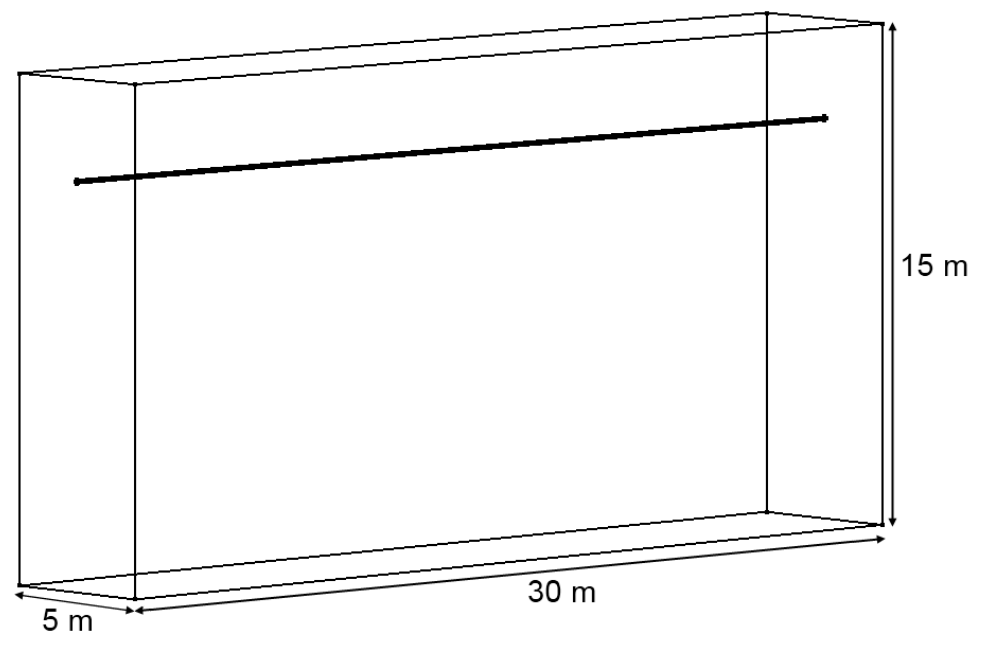

Figura 2. Instalação de Referência.

Para este estudo, sete dimensões definem o domínio do problema $\left(H, W, L, L_{0}, L_{1}\right.$, $D_{0}$ e $\left.D_{1}\right)$ e dois graus de liberdade que podem ser variados $\left(D_{1} / D_{0}\right.$ e $\left.L_{1} / L_{0}\right)$, conforme Figura 1. Manteve-se constante a profundidade do solo $(H)$, enquanto que a geometria varia somente em função da relação $L_{1} / L_{0}$ pois a relação $D_{1} / D_{0}$ será mantida constante $\left(D_{1} / D_{0}=1\right)$.

Como restrição do domínio têm-se o volume ocupado pelos dutos $\left(V_{D}\right)$, uma vez que o diâmetro dos dutos é mantido constante $\left(D_{0}=D_{1}=110 \mathrm{~mm}\right)$ e o comprimento total dos dutos também é mantido constante com $30 \mathrm{~m}$. A equação que representa $V_{D}$ é dada por:

$$
V_{D}=\frac{\pi}{4} L_{0} D_{0}^{2}+\frac{\pi}{2} L_{1} D_{1}^{2}
$$

Os valores de $L_{0}$ e $L_{1}$ são obtidos a partir da Equação 1 e dos valores de $D_{1} / D_{0}$ e $L_{1} / L_{0}$

Com relação à discretização espacial do domínio computacional, foi usado como referência o trabalho de Rodrigues et al. (2015). Porém, foi necessária a utilização de uma malha mais refinada devido à maior complexidade da geometria do TCSA em T. Sendo assim, um teste de convergência de malha foi realizado para o TCSA em T, utilizando o diâmetro $(D)$ dos dutos para definir o critério para o tamanho dos elementos do domínio. Definiu-se $D / 7$ e $3 \cdot D$, respectivamente, como tamanho das células computacionais do duto e do solo. Esse critério foi então usado na Instalação de Referência e o resultado foi comparado com os obtidos por Nunes (2015), verificando o modelo computacional. Em ambos os casos, foram utilizados volumes finitos tetraédricos. 


\section{MODELAGEM MATEMÁTICA}

A modelagem matemática utilizada para o estudo de TCSA foi fundamentada no princípio de conservação da massa, quantidade de movimento e energia.

Conforme Maliska (2010) e Versteeg e Malalasekera (2007), a equação de conservação da massa em sua forma geral pode ser utilizada tanto para escoamentos incompressíveis quanto para escoamentos compressíveis. Como no problema deste estudo o escoamento é incompressível, a equação de conservação da massa assume sua forma reduzida, sendo representada por:

$$
\frac{\partial \bar{v}_{i}}{\partial \bar{x}_{i}}=0
$$

onde: $v_{i}$ é o campo de velocidades na direção $i=1,2$ e $3(\mathrm{~m} / \mathrm{s}) ; x_{i}$ é a coordenada espacial na direção $i=1,2$ e $3(\mathrm{~m})$; e ( ) representa parâmetros de média no tempo.

Já o princípio fundamental da conservação da quantidade de movimento deve ser considerado para estudar o escoamento do ar no interior do duto. A equação da conservação da quantidade de movimento para um sistema não acelerado é dada por:

$$
\frac{\partial \bar{v}_{i}}{\partial t}+\frac{\partial\left(\bar{v}_{i} \bar{v}_{j}\right)}{\partial x_{j}}=-\frac{1}{\bar{\rho}} \frac{\partial \bar{\rho}}{\partial x_{j}} \delta_{i j}+\frac{\partial}{\partial x_{j}}\left[v\left(\frac{\partial \bar{v}_{i}}{\partial x_{j}}+\frac{\partial \bar{v}_{j}}{\partial x_{i}}\right)-\tau_{i j}\right]
$$

onde: $\rho$ é a massa específica do fluido $\left(\mathrm{kg} / \mathrm{m}^{3}\right) ; v$ é a viscosidade cinemática do $\operatorname{ar}\left(\mathrm{m}^{2} / \mathrm{s}\right)$; $p$ é a pressão $\left(\mathrm{N} / \mathrm{m}^{2}\right)$; $\delta_{i j}$ é o delta de Kronecker; e $t$ representa o domínio temporal (s).

Os termos $\tau_{i j}$ (tensor das tensões de Reynolds) surgem do processo de média temporal da equação de conservação da quantidade de movimento, sendo definidos por:

$$
\tau_{i j}=\overline{v_{l}^{\prime} v_{J}^{\prime}}
$$

Os problemas de TCSA envolvem transferência de energia térmica e por esse motivo a conservação da energia deve ser considerada. A equação da conservação da energia é dada por:

$$
\frac{\partial \bar{T}}{\partial t}+\frac{\partial}{\partial x_{j}}\left(\bar{v}_{j} \bar{T}\right)=\frac{\partial}{\partial x_{j}}\left[\alpha \frac{\partial \bar{T}}{\partial x_{j}}-q_{j}\right]
$$


sendo: $\alpha$ a difusividade térmica $\left(\mathrm{m}^{2} / \mathrm{s}\right)\left(\alpha=65,54 \cdot 10^{-8} \mathrm{~m}^{2} / \mathrm{s}\right)$; e $T$ a temperatura $(\mathrm{K})$. O termo $q_{j}$ (fluxo turbilhonar de energia) surge do processo de média temporal da equação da energia, sendo representado por:

$$
q_{j}=\overline{v_{j}^{\prime} T^{\prime}}
$$

Além disso, os escoamentos em TCSA são turbulentos, portanto, faz-se necessário a modelagem da turbulência. Neste estudo, foi realizada através do modelo $k-\varepsilon$ que, resumidamente, consiste na resolução de duas equações diferencias de transporte que representam a energia cinética turbulenta $(k)$ e a dissipação viscosa turbulenta $(\varepsilon)$.

\section{MODELAGEM NUMERICA}

A modelagem numérica do problema foi realizada utilizando os softwares GAMBIT e FLUENT. O GAMBIT foi utilizado na etapa de pré-processamento, na qual ocorre a construção do domínio computacional, a definição das condições de contorno e a discretização do domínio (geração da malha). Nas etapas de processamento e pósprocessamento foi utilizado o FLUENT, software baseado no Método dos Volumes Finitos (MVF) e que permite a modelagem de escoamento de fluidos e de transferência de calor em domínios computacionais complexos.

O esquema de advecção upwind foi utilizado para tratar os termos advectivos, uma vez que considera a direção do fluxo durante o cálculo das faces dos volumes de controle (PATANKAR, 1980). Além disso, o uso deste esquema elimina a existência de coeficientes negativos dos termos advectivos, garantindo a obtenção de uma solução livre de oscilações numéricas (MALISKA, 2010).

Para o acoplamento pressão-velocidade foi utilizado o modelo Coupled por ser indicado para escoamentos transientes quando a qualidade da malha é baixa ou quando são empregados grandes passos de tempo (FLUENT 6.3).

\section{RESULTADOS E DISCUSSÖES}

Nesta seção serão apresentadas e discutidas as diferentes configurações geométricas de instalações de TCSA com arranjo de dutos na forma de T.

As instalações com arranjo dos dutos em T foram definidas a partir de variações do grau de liberdade $L_{1} / L_{0}$ respeitando a distância previamente estabelecida entre os dutos e as paredes laterais do solo para que não houvesse influência das condições de contorno 
nos resultados obtidos, ou seja, valores de $L_{0}$ e $L_{1}$ assumidos são maiores ou iguais a $2 \mathrm{~m}$. Deste modo, obteve-se as 15 variações construtivas para a instalação que podem ser observadas na Figura 3.

As simulações realizadas para este trabalho representam um intervalo de tempo de 2 anos. O primeiro ano de simulação é necessário para que a temperatura do solo se estabilize, sendo então as análises realizadas com os dados do segundo ano de simulação. Adotou-se um passo de tempo de 3600 s e um máximo de 200 iterações para cada passo de tempo. Além disso, foi feito um monitoramento da temperatura nas saídas dos dutos a cada $6 \mathrm{~h}$, resultando em quatro registros de temperatura por dia.

Para a análise numérica das instalações considerou-se três parâmetros: o potencial térmico do TCSA, o volume de solo ocupado pela instalação e a perda de carga do escoamento.
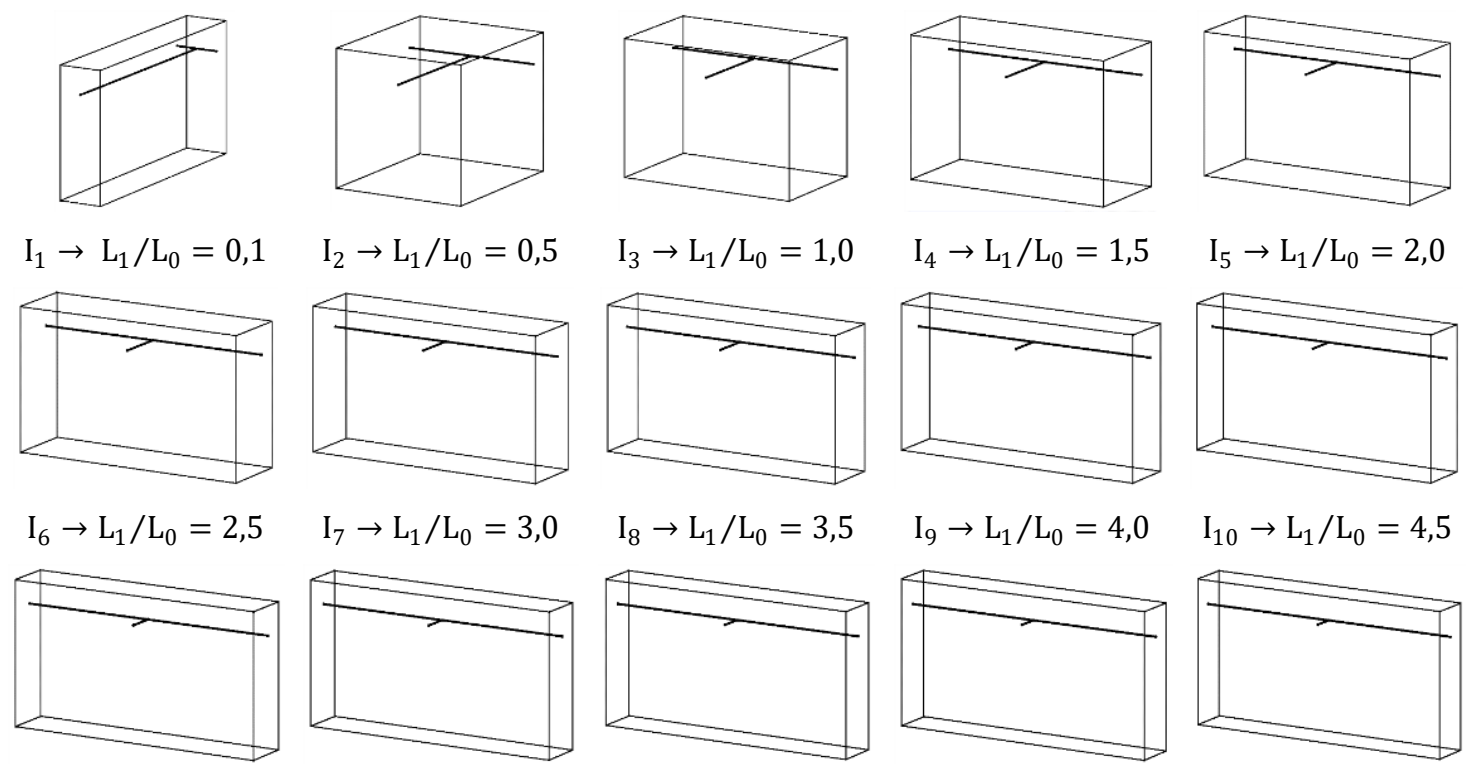

$\mathrm{I}_{11} \rightarrow \mathrm{L}_{1} / \mathrm{L}_{0}=5,0$

$\mathrm{I}_{12} \rightarrow \mathrm{L}_{1} / \mathrm{L}_{0}=5,5$

$\mathrm{I}_{13} \rightarrow \mathrm{L}_{1} / \mathrm{L}_{0}=6,0$

$\mathrm{I}_{14} \rightarrow \mathrm{L}_{1} / \mathrm{L}_{0}=6,5$

$\mathrm{I}_{15} \rightarrow \mathrm{L}_{1} / \mathrm{L}_{0}=7,0$

Figura 3. Configurações geométricas obtidas a partir da variação de $L_{1} / L_{0}$.

\subsection{Potencial Térmico}

O potencial térmico é calculado através da diferença de temperatura do ar entre a saída e a entrada do TCSA em relação ao número de registros da temperatura por dia (RODRIGUES, 2014):

$$
P T=\sum_{i=1}^{t}\left(\frac{\bar{T}_{s_{i}}-\bar{T}_{e_{i}}}{4 t}\right)
$$


onde: $P T$ é o potencial térmico médio $\left({ }^{\circ} \mathrm{C}\right)$ do TCSA; $\bar{T}_{S_{i}}$ é a temperatura média $\left({ }^{\circ} \mathrm{C}\right)$ na saída de ar dos dutos; $\bar{T}_{e_{i}}$ é a temperatura média $\left({ }^{\circ} \mathrm{C}\right)$ na entrada de ar dos dutos; e $i=$ $1,2, \ldots, t$, sendo to tempo (em dias) referente ao mês de análise.

A avaliação do potencial térmico das instalações de TCSA com configuração geométrica em T foi realizada em função do tempo durante o período de um ano.

Como é possível observar na Tabela 2, a Instalação 1 apresenta o maior potencial térmico de aquecimento e de resfriamento sendo, respectivamente, de 1,938ㄷ (em junho) e -8,025ㄷ (em dezembro). Ou seja, o potencial térmico é maximizado quando o arranjo de dutos na forma de $\mathrm{T}$ tem $L_{0} \gg L_{1}$, proporcionando a melhor troca de calor entre ar e solo. Esse comportamento pode ser explicado pela divisão do escoamento que ocorre na junção entre o trecho simples e o bifurcado.

Tabela 2. Potencial térmico das instalações.

\begin{tabular}{|c|c|c|c|}
\hline Instalação & L1/L0 & $\begin{array}{c}\text { Maior } P T\left({ }^{\circ} \mathrm{C}\right) \text { de aquecimento } \\
\text { (período anual) }\end{array}$ & $\begin{array}{l}\text { Maior } P T\left({ }^{\circ} \mathrm{C}\right) \text { de resfriamento } \\
\text { (período anual) }\end{array}$ \\
\hline$I_{R}$ & - & 1,906 & $-8,118$ \\
\hline$I_{1}$ & 0,1 & 1,938 & $-8,025$ \\
\hline $\mathrm{I}_{2}$ & 0,5 & 1,749 & $-7,788$ \\
\hline $\mathrm{I}_{3}$ & 1,0 & 1,728 & $-7,614$ \\
\hline $\mathrm{I}_{4}$ & 1,5 & 1,709 & $-7,473$ \\
\hline $\mathrm{I}_{5}$ & 2,0 & 1,708 & $-7,423$ \\
\hline $\mathrm{I}_{6}$ & 2,5 & 1,703 & $-7,352$ \\
\hline $\mathrm{I}_{7}$ & 3,0 & 1,715 & $-7,358$ \\
\hline $\mathrm{I}_{8}$ & 3,5 & 1,716 & $-7,300$ \\
\hline$l_{9}$ & 4,0 & 1,728 & $-7,291$ \\
\hline $\mathrm{I}_{10}$ & 4,5 & 1,737 & $-7,274$ \\
\hline $\mathrm{I}_{11}$ & 5,0 & 1,747 & $-7,259$ \\
\hline $\mathrm{I}_{12}$ & 5,5 & 1,749 & $-7,206$ \\
\hline $\mathrm{I}_{13}$ & 6,0 & 1,759 & $-7,199$ \\
\hline $\mathrm{I}_{14}$ & 6,5 & 1,768 & $-7,184$ \\
\hline $\mathrm{l}_{15}$ & 7,0 & 1,776 & $-7,161$ \\
\hline
\end{tabular}

Além disso, o potencial térmico de aquecimento da Instalação 1 é superior ao da Instalação Referência e quanto ao potencial térmico de resfriamento, a diferença apresentada é de apenas $0,093^{\circ} \mathrm{C}$. Cabe destacar que esses resultados correspondem apenas a uma saída do duto em T. Portanto, com um potencial térmico similar é possível atender duas edificações, enquanto que a Instalação Referência atende somente uma. 


\subsection{Volume de Solo Ocupado}

O volume de solo ocupado foi calculado a partir da configuração geométrica de cada instalação, uma vez que, exceto pela altura $(H)$ que foi mantida constante, o comprimento $(L)$ e a largura $(W)$ do solo variam conforme as alterações do grau de liberdade $L_{1} / L_{0}$ (ver Figura 3). O volume de solo ocupado por cada instalação é apresentado na Figura 4, sendo possível observar que a geometria correspondente à razão $L_{1} / L_{0}=7$ (Instalação 15) apresentou o menor volume de solo ocupado com $1,73 \times 10^{3} \mathrm{~m}^{3}$, representando uma redução da porção de solo de $55,18 \%$ se comparado à Instalação Referência. A redução do volume de solo viabiliza a utilização do TCSA no perímetro urbano, onde as dimensões dos terrenos são relativamente reduzidas.

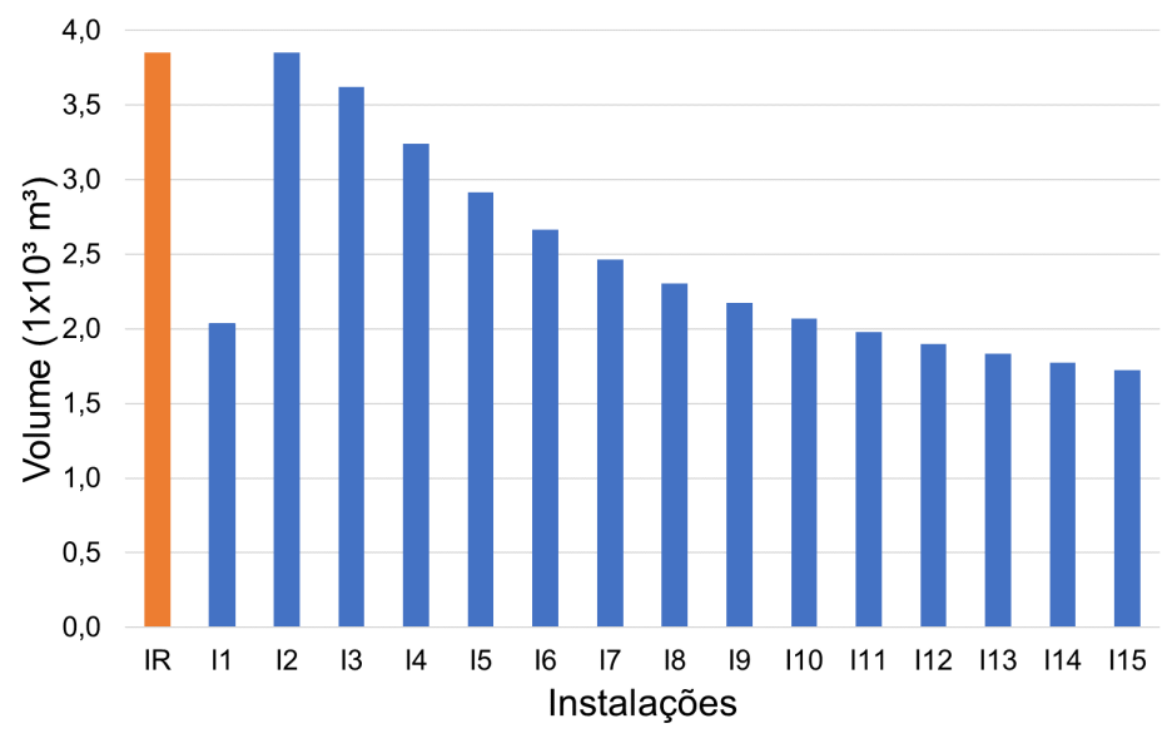

Figura 4. Comparação do volume de solo ocupado pelas instalações.

\subsection{Perda de Carga}

A perda de carga em escoamento turbulento forçado no interior de um duto compreende as perdas de carga distribuídas que ocorrem nos trechos cilíndricos longos do duto e as perdas de cargas localizadas que ocorrem nas descontinuidades existentes ao longo do duto, como por exemplo, mudanças de direção e de seção, presença de válvulas, variações de área e outras (BISTAFA, 2010).

A perda de carga total é determinada por:

$$
h=h_{f}+\sum h_{s}
$$


Sendo que $h_{f}$ é a perda de carga distribuída e $h_{s}$ é a perda de carga e são representadas, por:

$$
\begin{gathered}
h_{f}=f \cdot \frac{L}{D} \cdot \frac{v^{2}}{2 g} \\
h_{s}=K_{s} \cdot \frac{v^{2}}{2 g}
\end{gathered}
$$

onde $L$ é o comprimento da tubulação $(\mathrm{m}), D$ é o diâmetro do duto $(\mathrm{m}), v$ é a velocidade do escoamento $(\mathrm{m} / \mathrm{s})$ e $g$ é a aceleração da gravidade $\left(\mathrm{m} / \mathrm{s}^{3}\right)$. O coeficiente $f$ é o fator de atrito e pode ser calculado por uma correlação desenvolvida por Petukhov que possui a forma (DHAR, 2017):

$$
f=(0.79 \ln R e-1.64)^{-2}, \quad 10^{4}<R e<5 \times 10^{6}
$$

O coeficiente $K_{S}$ é determinado de acordo com o acessório que altera a direção do fluxo e para o caso de estudo em que há um T (com uma entrada e saída bilateral), 0 valor $K_{S}$ considerado é igual a 1,8 (BARRAL, 2018).

Na Figura 5, percebe-se a diminuição da perda de carga ao longo das variações na configuração geométrica das instalações de TCSA. Sob esse aspecto, a melhor geometria é a Instalação 15 que possui perda de carga de $1,79 \mathrm{~m}$, o que corresponde a uma diminuição na perda de carga de 53\% se comparada à Instalação Referência.

Além disso, a partir dos resultados obtidos, realizou-se um ajuste de curva para obter a equação característica do conjunto de dados. A curva em azul na Figura 5 representa com boa aproximação todas as instalações em T (ver Figura 3) analisadas nesse estudo.

A equação ajustada, a partir da Figura 5, é dada por:

$$
h=2,6279 \cdot\left(\frac{L_{1}}{L_{0}}\right)^{-0,213}
$$




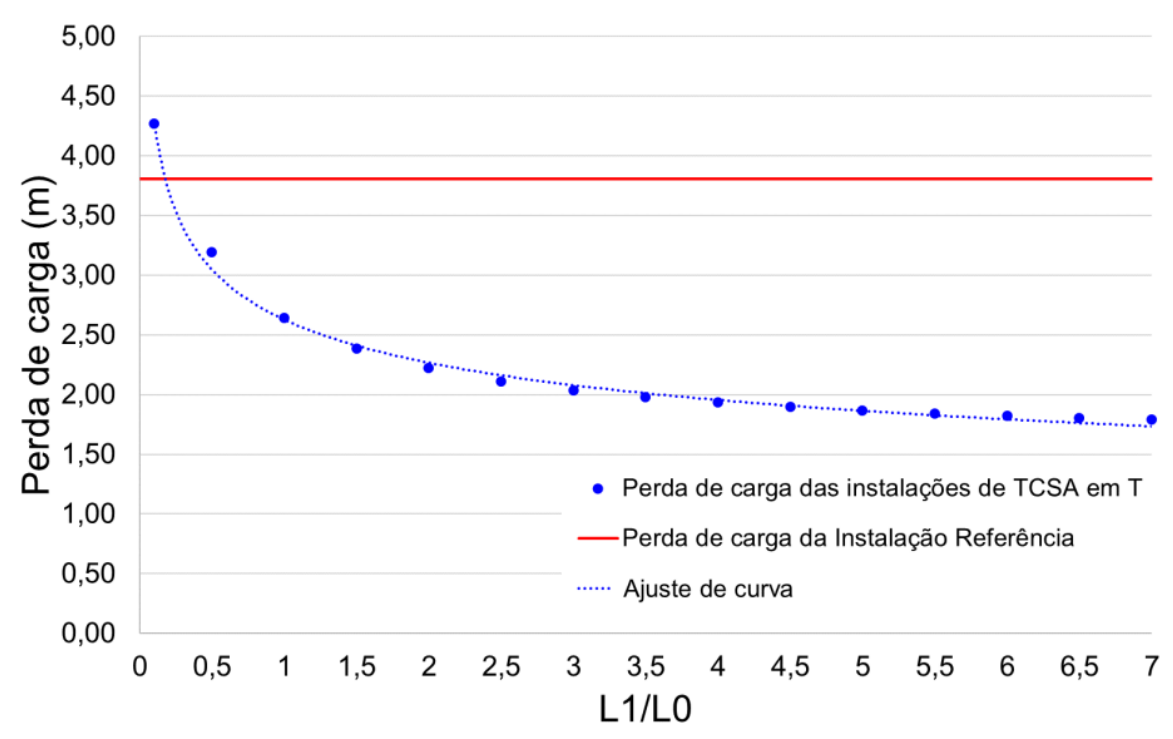

Figura 5. Perda de carga das instalações.

\subsection{Desempenho Global}

Para definir qual configuração geométrica possui melhor desempenho global, realizou-se uma análise vetorial a partir da normalização dos parâmetros anteriormente apresentados.

A normalização do potencial térmico médio é feita através da equação:

$$
P T_{N}=\frac{P T_{I}}{P T_{R}}
$$

sendo que $P T_{N}$ é o potencial térmico médio normalizado; $P T_{I}$ é o potencial térmico médio da instalação de análise; e $P T_{R}$ é o potencial térmico médio da Instalação Referência.

O potencial térmico da instalação com dutos em $\mathrm{T}$ deve ser igual ou superior ao da Instalação Referência, ou seja, $P T_{N} \geq 1$. No entanto, para que seja possível a comparação com os outros dois parâmetros, será adotado o valor de:

$$
\frac{1}{P T_{N}} \leq 1
$$

Já o volume de solo ocupado é normalizado por:

$$
V_{N}=\frac{V_{I}}{V_{R}}
$$

onde $V_{N}$ é o volume de solo normalizado; $V_{I}$ é o volume de solo ocupado pela instalação 
de análise; e $V_{R}$ é o volume de solo ocupado pela Instalação Referência.

Como este parâmetro deve ser minimizado, ou seja, busca-se reduzir a porção de solo ocupada pela instalação com dutos em T em relação à Instalação Referência, adotase $V_{N} \leq 1$.

Por último, têm-se a normalização da perda de carga que é representada por:

$$
h_{N}=\frac{h_{I}}{h_{R}}
$$

onde $h_{N}$ é a perda de carga normalizada; $h_{I}$ é a perda de carga da instalação que está sendo analisada; e $h_{R}$ é a perda de carga da Instalação Referência.

A perda de carga da instalação com arranjo de dutos em formato de $T$ também deve ser minimizada em relação à Instalação Referência, portanto, têm-se $h_{N} \leq 1$.

A partir da normalização dos parâmetros foi realizada uma análise vetorial para cada instalação. Para tal, buscou-se minimizar $1 / P T_{N}, V_{N}$ e $h_{N}$ de modo a encontrar o vetor de menor módulo em relação à origem do sistema de coordenadas. A Figura 6 mostra o módulo do vetor de cada instalação onde é possível observar que a Instalação 15 corresponde ao menor módulo, possuindo um valor 1,3246. Porém, fica evidente que as Instalações 10 até 15 possuem um comportamento muito semelhante quando se considera concomitantemente os parâmetros $1 / P T_{N}, V_{N}$ e $h_{N}$.

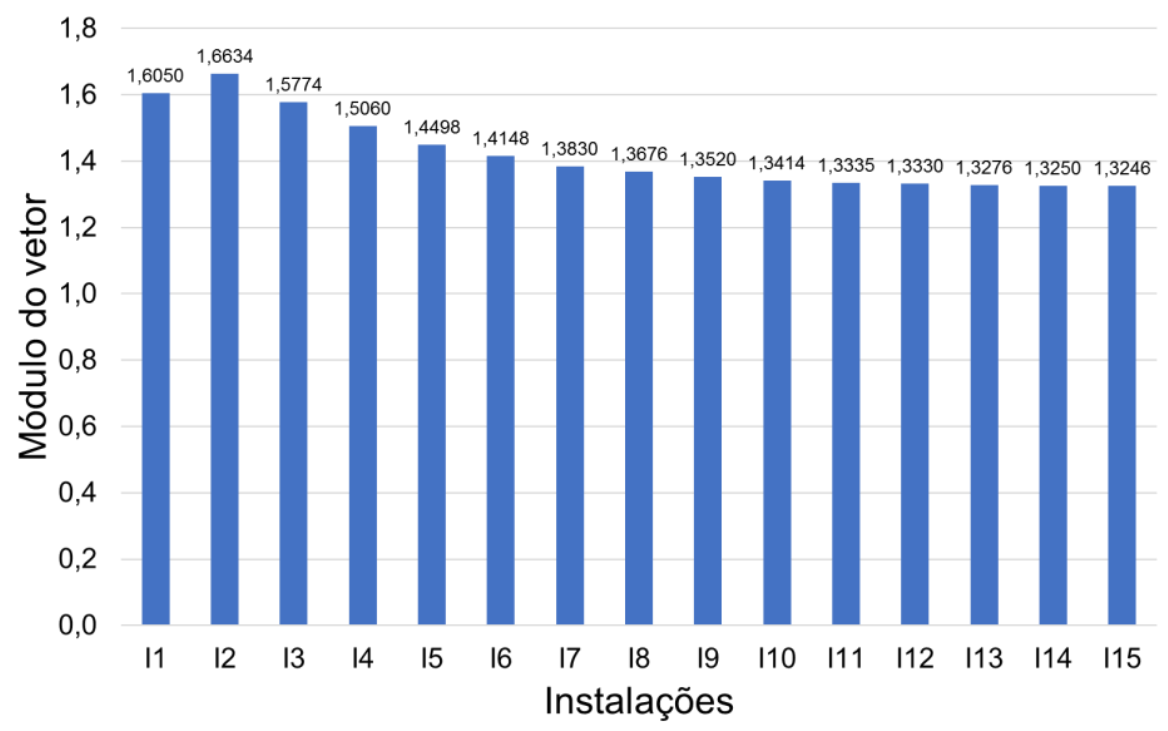

Figura 6. Módulo do vetor. 


\section{CONSIDERAÇOES FINAIS}

Neste trabalho, realizou-se um estudo numérico de instalações de TCSA com configuração geométrica na forma de $\mathrm{T}$, visando identificar a melhor geometria a partir da análise do volume de solo ocupado pelo dispositivo, seu potencial térmico e a perda de carga imposta ao escoamento do ar.

Através da associação do método Constructal Design e da modelagem computacional, os resultados numéricos obtidos indicam que a Instalação 1 apresenta 0 melhor desempenho térmico entre as instalações analisadas, inclusive com potencial térmico de aquecimento superior ao da Instalação Referência. Quanto aos demais parâmetros, comparados à Instalação Referência, a Instalação 15 apresentou uma redução do volume de solo ocupado de 55,18\% e uma diminuição na perda de carga de $53 \%$.

A partir destas análises foi possível concluir que a instalação que possui $L_{0} \gg L_{1}$, definida por $L_{1} / L_{0}=0,1$, maximiza o potencial térmico, enquanto que a que possui $L_{1} \gg$ $L_{0}$, definida por $L_{1} / L_{0}=7,0$, minimiza o volume de solo ocupado e a perda de carga do escoamento. Por fim, uma análise considerando esses três parâmetros simultaneamente, indicou que existem algumas configurações geométricas que possuem um desempenho superior, sendo a melhor configuração geométrica definida por $L_{1} / L_{0}=7,0$.

Como proposta de continuidade deste trabalho, é sugerida a investigação da influência do grau de liberdade $D_{1} / D_{0}$ no volume ocupado pelos dutos, no potencial térmico e na perda de carga.

\section{AGRADECIMENTOS}

Os autores agradecem ao Conselho Nacional de Desenvolvimento Científico e Tecnológico (CNPq), à Comissão de Aperfeiçoamento de Pessoal de Nível Superior (CAPES) e à Fundação de Amparo à Pesquisa do Estado do Rio Grande do Sul (FAPERGS) (Edital 02/2017 - PqG: 17/2551-0001 - 111-2) pelo apoio financeiro.

\section{REFERENCIAS}

AMANOWICZ, L. Influence of geometrical parameters on the flow characteristics of multi-pipe earth-to-air heat exchangers - experimental and CFD investigations. Applied Energy, n. 226, p. 849-861, 2018. 
BANSAL, V.; MISRA, R.; DAS AGRAWAL, G.; MATHUR, J. Performance analysis of earth-pipe-air heat exchanger for winter heating. Energy and Buildings, n. 41, p. 11511154, 2009.

BARRAL, M. F. Perda de carga. Disponível em: <http://www.esalq.usp.br/departamentos/leb/disciplinas/Fernando/leb472/Aula_7/Perda_d e_carga_Manuel\%20Barral.pdf> Acesso em: 20 ago. 2018

BEJAN, A. Constructal-theory network of conducting paths for cooling a heat generating volume. Journal Heat Mass Transfer, n. 40, p. 799-816, 1996.

BELATRACHE, D.; BENTOUBA, S.; BAUROUIS, M. Numerical analysis of earth air heat exchangers at operating conditions in arid climates. International Journal of Hydrogen Energy, n. 30, p. 1-7, 2017.

BENHAMMOU, M.; DRAOUI, B. Parametric study on thermal performance of earth-toair heat exchanger used for cooling of buildings. Renewable and Sustainable Energy Reviews, n. 44, p. 348-355, 2015.

BISONIYA, T. S.; KUMAR, A.; BAREDAR, P. Experimental and analytical studies of earth-air heat exchanger (EAHE) systems in India: A review. Renewable and Sustainable Energy Reviews, n. 19, p. 238-246, 2013.

BISTAFA, S. R. Mecânica dos fluidos: noções e aplicações. São Paulo: Blucher, 2010.

BRUM, R. S.; ROCHA, L. A. O.; VAZ, J.; DOS SANTOS, E. D.; ISOLDI, L. A. Development of Simplified Numerical Model for Evaluation of the Influence of SoilAir Heat Exchanger Installation Depth over Its Thermal Potential. International Journal of Advanced Renewable Energy Research, v. 1, p. 505-514, 2012.

BRUM, R. Teoria Construtal e desempenho térmico de trocadores de calor solo-ar. Tese. Universidade Federal do Rio Grande do Sul (UFRGS), 2016.

DHAR, P. L. Thermal System Design and Simulation. Elsevier Ltd, 2017.

FLUENT 6.3. Documentation Manual. Disponível em: <https://www.sharcnet.ca/Software/Fluent6/html/ug/node998.htm> Acesso em: 29 ago. 2018.

MALISKA, C. R. Transferência de calor e mecânica dos fluidos computacionais. 2 ed. LTC - Livros Técnicos e Científicos Editora S.A., Rio de Janeiro, 2010.

NIU, F.; YU, Y.; YU, D.; LI, H. Heat and mass transfer performance analysis and cooling capacity prediction of earth to air heat exchanger. Applied Energy, n. 137, p. 211-221, 2015.

NUNES, B. R. Modelagem computacional aplicada ao estudo de trocador de calor solo-ar com configuração geométrica complexa. Dissertação. Universidade Federal do Rio Grande (FURG), 2015. 
PATANKAR, S. Numerical heat transfer and fluid flow. Hemisphere Corporation, 1980.

RODRIGUES, M. Modelagem computacional aplicada à melhoria do desempenho térmico de trocador de calor solo-ar através do método Constructal Design. Dissertação. Universidade Federal do Rio Grande (FURG), 2014.

RODRIGUES, M.; BRUM, R.; VAZ, J.; ROCHA, L. A. O.; DOS SANTOS, E. D.; ISOLDI, L. $A$. Numerical investigation about the improvement of the thermal potential of an earth-air heat exchanger (EAHE) employing the Constructal Design method. Renewable Energy, n. 80, p. 538-551, 2015.

SEN, Z. Solar Energy Fundamentals and Modeling Techniques: Atmosphere, Enviroment, Climate Change and Rewable Energy. Springer, 2008.

TRZASKI, A.; ZAWADA, B. The influence of environmental and geometrical factors on air-ground tube heat exchanger energy efficiency. Building and Environment, $\mathrm{n}$. 46, p. 1436-1444, 2011.

VAZ, J. Estudo experimental e numérico sobre o uso do solo como reservatório de energia para o aquecimento e resfriamento de ambientes edificados. Tese. Universidade Federal do Rio Grande do Sul (UFRGS), 2011.

VAZ, J.; SATTLER, M. A.; DOS SANTOS, E. D.; ISOLDI, L. A. Experimental and numerical analysis of an earth-air heat exchanger. Energy and Buildings, 43, 24762482, 2011.

VAZ, J.; SATTLER, M. A.; DOS SANTOS, E. D.; ISOLDI, L. A. An experimental study on the use of Earth-Air Heat Exchangers (EAHE). Energy and Buildings, 72, 122-131, 2014.

VERSTEEG, H. K.; MALALASEKERA, W. An Introduction to Computational Fluid Dynamics: The Finite Volume Method. England Pearson Education Limited, second edition, 2007. 